\title{
Les expressions idiomatiques dans le journal $L e$ Monde diplomatique : analyse des valeurs expressives et contextuelles des traductions français-croate
}

\author{
Larisa Grčić Simeunovic ${ }^{1, *}$, Matea Krpina $^{1}$, et Mojca Pecman $^{2}$ \\ ${ }^{1}$ Université de Zadar, Département d'études françaises et francophones, 1 rue M. Pavlinovića, 23000 \\ Zadar, Croatie \\ ${ }^{2}$ Université de Paris, CLILLAC-ARP, F-75013 Paris, France
}

Résumé. La fonction des expressions idiomatiques, en tant qu'unités de la langue servant à exprimer des images de la réalité correspondant à des situations dites stéréotypes et clichés, leur accorde une force expressive qui fait d'elles l'allié privilégié des journalistes. Les expressions idiomatiques sont des lexicalisations de valeurs d'une langue-culture et souvent elles se prêtent difficilement à une traduction dans une langue cible avec les mêmes valeurs. Cette étude s'appuie sur la notion de valeur pour distinguer la double valeur des expressions idiomatiques : leur valeur expressive (qui leur accorde une place spécifique dans le système de la langue) et leur valeur contextuelle (leur sens dans le discours). Elle a été réalisée à l'aide $\mathrm{du}$ corpus parallèle franco-croate du journal Le Monde diplomatique, qui s'inscrit dans des contextes culturels et politiques spécifiques et sousentend une approche interculturelle de la traduction. Les résultats de l'étude portent sur l'analyse des stratégies employées pour traduire des expressions idiomatiques avec le but de démontrer dans quelle mesure la difficulté qu'elles présentent amène le traducteur à se concentrer soit sur leur valeur expressive dans le système de la langue source soit sur leur valeur contextuelle exprimée dans le discours. Les analyses présentées dans cette étude se focalisent, par conséquent, sur l'analyse des rapports entre les structures linguistiques et discursives des expressions idiomatiques dans la version bilingue français-croate, en tenant compte de leurs aspects dénotatifs et connotatifs.

\begin{abstract}
The idiomatic expressions in the journal Le Monde diplomatique: a study of the expressive and contextual values in French -Croatian translations. The idiomatic expressions are specific type of language units that convey images of reality, corresponding to so-called stereotyped situations and clichés. This status gives them an expressive force of which the journalists are particularly fond. Idiomatic expressions are the lexicalizations of language-culture values and the transfer of those values into the target language (TL) is often hampered by the absence of an expression containing the same values in the TL. In this study, we use the notion of value to distinguish the double dimension of the idiomatic expressions: their expressive value (the value conveyed in the language) and their contextual value (the meaning expressed in the speech). To achieve our goal, we focus on the Franco-Croatian parallel corpus Le Monde diplomatique, which reflects specific cultural and political contexts and implies an intercultural approach to translation. The aim of this study is to analyse the translation strategies when dealing with idiomatic expressions, and to evaluate to what extent these strategies fail to transfer
\end{abstract}

\footnotetext{
*Corresponding author : lgrcic@unizd.hr
} 
both values of idiomatic expressions, expressive and contextual, and force the translator to choose between one of them. To achieve this analysis, the paper examines the relationship between linguistic and discursive patterns at stake in these expressions by taking into account the denotative and connotative aspects of the language.

\section{Introduction}

Les expressions idiomatiques (EI) sont des unités de langue qui servent à exprimer des images de la réalité et correspondent à des situations dites stéréotypes et clichés. Le recours aux images, sur la base du mécanisme de métaphore, leur accorde une force expressive qui fait d'elles l'allié privilégié des journalistes. Le discours journalistique est caractérisé par l'intention d'agir sur ses lecteurs et, en conséquence, par la recherche de l'expressivité lors de la transmission du message. Les expressions idiomatiques permettent de moduler l'expressivité du message en la renforçant. Nous partons ainsi du postulat qu'une EI comme faire des gros titres constitue un choix linguistique qui permet de renforcer davantage un message que ne le ferait une structure sans image et qui ne relève pas du fonds idiomatique de la langue, de type constituer le sujet abordé dans tous les journaux. En tant qu'allusion à des images déjà connues par le récepteur-source, souvent les EI se refusent à un transfert facile dans la langue cible avec les mêmes valeurs. Les expressions idiomatiques sont la lexicalisation de la perception d'une langue-culture et leur traduction nécessite une analyse fine des valeurs associées à ces dernières.

Pour cette étude, nous avons fait appel à la notion de valeur pour distinguer la double valeur des expressions idiomatiques : leur valeur expressive et leur valeur contextuelle. La première désigne leur valeur dans le système de la langue, la seconde leur valeur dans le discours. La notion de valeur, introduite par F. de Saussure (1969), est l'une des notions clés de la linguistique contrastive et de la traductologie. Ainsi, il est possible de rapprocher la dualité entre ces deux valeurs à l'opposition entre langue et parole (ou entre langue et discours). Néanmoins, la valeur expressive peut également trouver écho dans la conception de l'expressivité par Bally. Comme le remarque Curea (2008: 924-925), la notion d'expressivité selon Bally repose essentiellement sur l'idée de l'implicite. Pour expliquer sa conception du mécanisme de l'expressivité, Bally a recours à deux notions supplémentaires, l'impression sensorielle et la représentation imaginative : "Le langage, intellectuel dans sa racine, ne peut traduire l'émotion qu'en la transposant par le jeu d'associations implicites. (...) les associations s'attachent soit au signifiant, de manière à en faire jaillir une impression sensorielle, soit au signifié, de manière à transformer le concept en représentation imaginative. L'une et l'autre catégorie d'associations se chargent d'expressivité dans la mesure où la perception sensorielle ou la représentation imaginative concorde avec le contenu émotif de la pensée. " (Bally 1926: 83). Un exemple d'association s'attachant au signifiant est fourni par les onomatopées (ex. gazouiller, caracoler, grignoter, cliquetis). Dans la catégorie d'associations s'attachant au signifié, Bally place essentiellement les images, les figures ou les tropes (ex. un clou dans la dent, c'est une forte tête, la ville est en rumeur, ses yeux lancent des éclairs, le vent mugit).

En linguistique contrastive, les similarités et les différences entre les systèmes linguistiques sont analysées au niveau de la correspondance mais les correspondances linguistiques n'égalent pas toujours les équivalences discursives, ni inversement. En traduction, la valeur expressive présuppose une recherche complexe, aussi bien dans les systèmes de la langue source que dans ceux de la langue cible. L'individualité unique de chaque acte expressif exige du traducteur de tenir compte du contexte discursif tout en respectant la valeur expressive du système source. Dans le cas des expressions idiomatiques, la valeur expressive est donc due à la "représentation imaginative » du 
contenu sémantique véhiculé par l'expression qui exige du traducteur qu'il porte son attention sur la matérialité linguistique de l'expression autant que sur son sens au moment de l'actualisation dans le discours. En effet, les expressions imagées reposent sur la notion de trope et renferment une valeur due à ce premier transfert de sens, transfert métaphorique du sens propre au sens imagé. D'une part, cette valeur peut se révéler essentielle pour la transmission du message, comme dans le cas du discours journalistique. D'autre part, la traduction de la valeur contextuelle se doit de prendre en compte les critères pragmatiques.

En comparant nos deux versions du Monde diplomatique, en français et en croate, nous avons cherché les points de convergence et de divergence présents au niveau des expressions imagées, à savoir comment leurs valeurs expressives et contextuelles ont été retransmises du français au croate. Dans la presse journalistique, le choix du journaliste se cache derrière chaque mot et c'est pourquoi il est important que le traducteur ne se contente pas de recourir à une simple mise à plat sémantique, ou déverbalisation du contenant, pour n'en traduire que le contenu. Derrière chaque rédaction journalistique, il existe le choix du journaliste d'opter pour une formulation implicite ou explicite, de retenir ou d'exclure des informations liées au sujet traité, etc. Le traducteur, lorsqu'il traduit, se doit lui aussi de faire des choix. De plus, notre corpus s'inscrit dans différents contextes culturels et politiques et sous-entend une approche traductologique interculturelle (Ladmiral et Lipiansky 2015). Bien que le traducteur doive sauvegarder les valeurs contextuelles en langue cible, il ne peut se permettre de négliger la valeur des signifiants en langue source. Pour analyser cette problématique, nous nous sommes concentrées sur l'analyse des expressions idiomatiques imagées extraites du corpus du Monde diplomatique bilingue français-croate pour illustrer notre propos.

Les sections qui suivent présentent d'abord notre cadre théorique, puis notre méthode de dépouillement du corpus et, au final, les résultats de nos analyses.

\section{Idiomaticité à l'épreuve de la traduction : cadres théoriques et méthodologiques}

Avant de rappeler les problèmes de traduction posés par les éléments idiomatiques du langage, nous discutons du rôle des expressions idiomatiques dans le discours journalistique.

\subsection{Du rôle des expressions idiomatiques dans le discours journalistique}

Tout d'abord, la première fonction des expressions idiomatiques est d'exprimer les images de la réalité (c'est pourquoi, par ailleurs, elles ne sont pas présentes avec la même fréquence dans deux langues différentes). Dans un deuxième temps, les expressions idiomatiques représentent des situations dites stéréotypes et clichés. Selon Gambier (2006), les textes de presse se servent de cadres de référence ou de représentations stéréotypes de situations spécifiques permettant de rapprocher les faits (économiques, politiques, sociétaux, etc. ; nationaux ou internationaux) relayés au lectorat. Les scénarios stéréotypes ou les routines contiennent des connaissances attendues par les interlocuteurs. En cadrant le discours des médias, les journalistes créent un certain contexte, selon lequel le public est censé interpréter le message.

Puisque l'intention des journalistes est d'agir sur les lecteurs, ils s'engagent dans leur expressivité. Selon Bally (1926), l'expressivité consiste à modifier « l'expression existante en quantité ou en qualité (grossissement, rénovation, déformation) ». Par ailleurs, et toujours selon Bally (1926: 21), «nous interprétons les évènements de la réalité selon la valeur qu'elles représentent pour nous ou pour d'autres individus dont la vie est liée à la nôtre ». Par conséquent, le travail des journalistes, qui cherchent à interpréter la réalité, consisterait nécessairement en une transmission de ces valeurs partagées par la 
communauté de locuteurs, à laquelle ils s'adressent et dont ils font partie. Pour la transmission de ces valeurs, ils utilisent différentes tournures stylistiques, dont les expressions idiomatiques figées, pour accentuer l'expressivité du message. C'est la raison pour laquelle nous avons concentré notre étude sur ces expressions, étant donné qu'elles permettent de moduler l'expressivité du message.

\subsection{Traduction des expressions idiomatiques}

Quels sont les enjeux de la traduction des expressions idiomatiques ? Différents auteurs se sont penchés sur cette problématique : on peut citer les études de Th. Ben Amor (2008), Ch. Durieux (2008), Y. Haquin (2015), E. Le Bel (2006), J. Maillot (1984), S. Mejri (1997, 2008), G. Misri (1990), C. M. Xatara (2002) entre autres. Les expressions idiomatiques appartiennent à la langue au sens saussurien du terme, mais aussi au discours. Notre objectif a donc été de souligner l'importance du repérage de ces unités idiomatiques porteuses d'un sens différent du celui des mots qui les composent, afin de les analyser et pouvoir en réexprimer le sens (traduire) de façon adéquate lors du transfert culturel.

Le problème que représentent les expressions idiomatiques du point de vue de la traduction réside dans le fait qu'elles font allusion à une image déjà connue au récepteursource. Afin de traduire les expressions idiomatiques, le traducteur ne doit pas recourir à une simple recherche des correspondances linguistiques. Cette situation exige de lui la recherche d'une équivalence linguistique et contextuelle, et si le système cible ne lui permet pas de trouver une unité de traduction comportant ces deux valeurs à la fois, il devra compenser, c'est-à-dire réécrire afin de susciter les mêmes effets du texte de départ dans le texte d'arrivée (cf. Misri 1990). Le traducteur se confronte donc à deux niveaux de sens : le premier niveau est celui du système linguistique (ce que nous appelons la valeur expressive) et le deuxième niveau est celui du discours ou du texte (ce que nous appelons la valeur contextuelle). En effet, ce dernier semble être mise de côté afin de faire émerger le vouloir dire caché derrière les mots. Or, l'image véhiculée dans les expressions idiomatiques n'est jamais complètement détachée de son sens. C'est là que réside toute la difficulté pour les traducteurs. En reprenant Mejri (2008 : 244), la traduction des séquences figées « est une négociation permanente entre forme et contenu».

$\mathrm{Au}$ cours de la recherche de correspondances interlinguistiques des expressions idiomatiques, le traducteur se heurte à un manque de réversibilité étant donné qu'il n'y a pas de bijection automatique entre deux langues (Durieux 2006). Cela est dû aux différences de capacités expressives de chaque langue qui influencent la création des expressions idiomatiques dans le code de la langue. Puisque chaque système organise, combine et oppose les signes à sa propre façon, le traducteur est obligé de surpasser la traduction « littérale » et de se concentrer sur le sens subjectif et affectif. Par conséquent, pour traduire des expressions idiomatiques, il ne suffit pas uniquement de faire appel à des connaissances linguistiques mais aussi à des connaissances extralinguistiques et pragmatiques.

Deux interrogations principales s'imposent lors de la traduction des expressions idiomatiques : faut-il avoir recours à la déverbalisation, c'est-à-dire à la mise à plat du contenu sémantique de l'expression afin de n'en recueillir que le sens en contexte, ou bien plutôt préférer une approche qui se voudrait à la recherche d'une équivalence plus « fidèle » ou du moins respectueuse de la lettre. Cette dernière prend en considération les signes, en évitant de les traiter uniquement en tant que simple " coquille » superflue du sens qu'elle véhicule. La première approche a comme souci essentiel celui de la clarté dans le transfert de sens. Elle estime que le texte cible doit être compréhensible par le lectorat d'accueil et elle puise ses racines dans la théorie interprétative et la théorie du sens de Seleskovitch et Lederer (1984). Les deux théories accordent la primauté au « vouloir dire » sans s'attarder à ce que nous osons appeler la «manière de dire ». La seconde approche, en revanche, souligne l'importance du fond et de la forme, considérées indissociables. En effet, le 
système linguistique englobe des signes qui se construisent et s'actualisent en discours sur deux niveaux : celui du contenu de l'expression, du fond, et de la forme. Si la communication monolingue présuppose une interprétation, une actualisation des signifiants et des signifiés (unités de sens) du système dans le discours, la traduction exige du traducteur un double défi qui consiste à chercher les signes appropriés dans le système linguistique cible ainsi que de les actualiser selon le sens qu'ils doivent véhiculer. De ce fait, les unités linguistiques du système deviennent les unités de traduction dans le discours.

Les difficultés de traduction des expressions idiomatiques expliquent qu'un volet important des études s'inscrivant dans ce champ de recherche porte sur la construction des dictionnaires ou des répertoires des expressions, dont le but est d'offrir les ressources nécessaires pour la traduction (cf. les travaux de M. M. Dubois (1973), A. M. Laurian (2004), A. Rey et S. Chantreau (1994), H. Zinglé et M. L. Brobeck-Zinglé (2003) entre autres).

Même à l'aube des systèmes de traduction automatique à architecture neuronale, la traduction des expressions idiomatiques demeure un défi (cf. Vaguer 2011). Selon Burlot et Yvon (2017), les nouvelles architectures des systèmes de traduction automatique semblent capables de mieux « détecter (dans la langue source) et de modéliser (dans la langue cible) des dépendances entre mots distants et ainsi de mieux restituer des associations lexicales (collocations, expressions idiomatiques) ». On peut donc supposer que l'AI continuera à nous apporter une aide en traduction de plus en plus précieuse. Néanmoins, les systèmes de traduction automatique à architecture neuronale pour la langue croate sont encore relativement rares (cf. Arčan 2018, Ljubešić et al. 2010) et les tests de leur performance menée par les linguistes ne sont pas encore disponibles.

\section{Méthodologie}

Dans cette section nous présentons le corpus du Monde diplomatique en version bilingue français-croate et la procédure de sélection des expressions idiomatiques en vue de leur analyse.

\subsection{Le Monde Diplomatique : un corpus parallèle franco-croate du discours journalistique}

Pour réaliser notre recherche, nous nous sommes penchées sur un corpus parallèle francocroate du journal Le Monde diplomatique (LMD) dont les textes abordent des sujets actuels à thématiques politiques, économiques et culturelles. Bien que certaines théories linguistiques de la traduction, et plus particulièrement les pratiques d'analyse de la traduction, prétendent que tout texte peut se ramener à une séquentialité de phrases, l'approche pragmatique de la traduction (cf. Froeliger 2013) démontre la nécessité de prendre en compte la visée du discours et son élément central : le lecteur cible. L'exemple de LMD démontre bien que le texte ne peut être considéré de manière isolée, c'est-à-dire indépendamment du lecteur.

Le Monde diplomatique $e^{i}$ est un journal destiné à un lectorat cherchant des informations et des analyses concernant le monde entier. En 2018, ce journal comptait 29 éditions internationales en 18 langues ${ }^{\mathrm{ii}}$. En 2020, ces chiffres ont été portés à 31 éditions et à 22 langues. Le style journalistique se veut littéraire et les informations et les analyses relayées sont relatées d'une manière singulière. Une des caractéristiques du discours journalistique français est, en effet, de présenter un style assez imagé en recourant de façon assez répétitive à des expressions idiomatiques (cf. Charaudeau 2005).

Les textes de LMD doivent avoir les mêmes objectifs pour le lectorat source et le lectorat cible. Il s'agit d'un journal qui regroupe des textes ayant une utilité fonctionnelle 
qui va au-delà de la simple transmission d'informations. Le but est de susciter une réflexion chez son lectorat. Le Monde diplomatique est un journal qui cible des lecteurs avec un certain bagage de connaissances en amont. Ces lecteurs ont des attentes lorsqu'ils prennent le journal entre leurs mains, attentes auxquelles le LMD cherche à répondre. Les journalistes ont, en effet, tendance à créer du sens "en plus » grâce aux processus langagières et linguistiques permis par le français, et les expressions idiomatiques jouent un rôle important dans la création de ces sens « supplémentaires".

Puisque LMD traite des faits contemporains en provenance du monde entier, il est nécessaire d'adapter le niveau expressif et contextuel au lectorat cible. Il arrive pour cela que les traductions soient rédigées par le rédacteur en chef. Ce dernier évalue la traduction en tenant compte de la valeur contextuelle du texte. Cette adaptation est une forme d'adéquation qui laisse aux rédacteurs une certaine liberté en ce qui concerne la transmission du contenu et du style. Selon Gambier (2006), les deux versions localisées exprimées en différents systèmes langagiers recouvrent aussi différentes valeurs et idées culturelles, ainsi que les systèmes de catégorisation.

\subsection{Dépouillement du corpus}

Le dépouillement du corpus consistait en une analyse manuelle des articles de LMD qui nous a permis de relever les expressions idiomatiques dans le texte source et leurs équivalences dans le texte cible. Nous avons examiné les articles de trois numéros du LMD ( 754,755 et 756 de la version française et les numéros 49,50 et 51 de la version croate) publiés entre janvier et mars 2017.

Le critère de sélection, appliqué dans cette étude, consistait à choisir les locutions verbales (semi-) figées métaphoriques qui ont fait l'objet de nombreuses études en phraséologie française. On peut citer I. Gonzales Rey (2002), P. Mogorrón Huerta (2008), C. Vaguer (2011), parmi autres.

Afin de sélectionner ces expressions ciblées par notre étude, nous avons dû exclure tous les autres types d'expressions polylexicales. Le but de notre article n'est pas de discuter les typologies des diverses structures polylexicales idiomatiques, abordé dans nombreux travaux (voir par exemple R. Gläser (1988) et M. Benson et al. (1997) pour l'anglais dans le contexte de la langue générale, G. Gross (1996) pour le français dans le contexte de la langue générale ou M. P. Jacques et A. Tutin (2017) dans le contexte des sciences humaines, J. Jernej (1992) et D. Sesar (1998) pour la langue croate, et M. Pecman (2007) pour une approche contrastive français-anglais-croate de la classification des unités phraséologiques). Rappelons néanmoins que nous nous sommes appuyées sur ces typologies connues pour annoter les expressions idiomatiques.

Le Tableau 1 montre les diverses unités phraséologiques potentielles dans deux extraits de LMD en version bilingue français-croate. Lors du dépouillement du corpus, nous n'avons pas retenu les expressions figée ou locutions non imagées, ni les collocations ou toutes autres structures idiomatique marquées par le figement mais ne reposant pas sur des images à force expressive (ex. dans l'opinion, de près ou de loin, jouer un rôle, faits divers horrifiques, infractions imputables, assumer la charge, maîtriser l'action, mettre dans une position difficile, comme l'a relevé), ni les unités polylexicales qui sont des créations (ex. revêtir ses habits favoris, submerger par une vague qui ne sont pas des expressions de type catachrèse, c'est-à-dire des expressions imagées ou métaphoriques entrées dans l'usage courant). A l'inverse, nous avons retenu les expressions idiomatiques polylexicales imagées qui font partie de l'usage courant et dont la structure implique un noyau verbal (ex. peser lourd, faire des gros titres, noyer sous les flots, déverser des insanités, bafouer la souveraineté, se présenter sur un plateau d'argent), parmi lesquelles nous avons sélectionné un échantillon pour les soumettre à l'analyse (en l'occurrence peser lourd, faire des gros titres, se présenter sur un plateau d'argent et noyer sous les flots). 
Le Tableau 1 montre deux extraits du corpus parallèle avec les annotations effectuées : les différents types d'expressions idiomatiques et collocations sont encadrées, les éléments imagés sont en rouge, les exemples sélectionnés et analysés sont soulignés.

Tableau 1. Exemple des extraits du corpus parallèle avec les annotations des expressions idiomatiques

\begin{tabular}{|c|c|c|}
\hline & Français & Traduction en croate \\
\hline $\begin{array}{l}\text { Extrait } \\
1\end{array}$ & $\begin{array}{l}\text { [Dans l'opinion], la crainte du } \\
\text { terrorisme [joue toutefois un } \\
\text { rôle] secondaire par rapport au } \\
\text { [spectre de la "criminalité] } \\
\text { étrangère ». L'exploitation de } \\
\text { plusieurs [faits divers } \\
\text { horrifiques], comme les } \\
\text { agressions sexuelles massives } \\
\text { perpétrées à Cologne le soir du } 31 \\
\text { décembre 2015, le viol et } \\
\text { l'assassinat d'une étudiante par un } \\
\text { réfugié afghan à Fribourg en } \\
\text { octobre 2016, ou encore } \\
\text { l'arrestation en décembre d'un } \\
\text { groupe de mineurs syriens } \\
\text { accusés d'avoir jeté un papier en } \\
\text { feu sur un sans-abri dans le métro } \\
\text { berlinois, [pèse lourd] dans } \\
\text { l'imaginaire collectif. Peu importe } \\
\text { si les statistiques de l'Office } \\
\text { fédéral de la police criminelle } \\
\text { (BKA) indiquent que les } \\
\text { étrangers ne commettent pas } \\
\text { davantage de crimes et de délits } \\
\text { que les Allemands et que les } \\
\text { [infractions qui leur sont } \\
\text { imputables] tendent même à } \\
\text { diminuer : cela [ne fera pas les } \\
\text { gros titres]. [Noyés sous les } \\
\text { flots] [d'insanités racistes qui se } \\
\text { déversent] désormais sur } \\
\text { Internet, les sites de plusieurs } \\
\text { journaux ont fermé les } \\
\text { commentaires sous les articles } \\
\text { consacrés [de près ou de loin] à } \\
\text { la question des réfugiés. }\end{array}$ & $\begin{array}{l}\text { [U javnom mišljenju], } \\
\text { strah od terorizma ipak igra } \\
\text { manju ulogu od [tvrdnji o } \\
\text { « kriminalitetu] stranaca ». } \\
\text { Senzacionalistička eksplo- } \\
\text { atacija nekoliko [različitih } \\
\text { zastrašujućih incidenata], } \\
\text { poput seksualnih napada u } \\
\text { Kölnu 31. prosinca 2015., } \\
\text { silovanja i ubojstva stu- } \\
\text { dentice od strane izbjeglice } \\
\text { afganistanskog podrijetla u } \\
\text { Freiburgu u listopadu 2016. } \\
\text { ili pak uhićenja skupine } \\
\text { maloljetnih Sirijaca optu- } \\
\text { ženih za napad na } \\
\text { beskućnika u berlinskom } \\
\text { metrou u prosincu, [ostavila } \\
\text { je utjecaja]. Malo vrijedi } \\
\text { što statistike Saveznog } \\
\text { ureda kriminalističke } \\
\text { policije (BKA) pokazuju da } \\
\text { stranci ne čine više zločina i } \\
\text { prekršaja od Nijemaca te da } \\
\text { su [prekršaji koji im se } \\
\text { pripisuju] u padu. Takve } \\
\text { vijesti [ne dospijevaju na } \\
\text { naslovnice]. [Preplavljene] } \\
\text { [rasističkim besmislicama } \\
\text { koje se šre] internetom, } \\
\text { neki su mediji na svojim } \\
\text { stranicama morali onemo- } \\
\text { gućiti komentiranje članaka } \\
\text { koji su posvećeni pitanju } \\
\text { izbjeglica. }\end{array}$ \\
\hline $\begin{array}{l}\text { Source } \\
1\end{array}$ & $\begin{array}{l}\text { Le Monde diplomatique, mars } \\
2017 \text {, «Un grand élan de } \\
\text { solidarité, mais une xénophobie } \\
\text { qui progresse; L'Allemagne et les } \\
\text { réfugiés, deux ans après » par } \\
\text { Olivier Cyran, pages 12-13 }\end{array}$ & $\begin{array}{l}\text { Le Monde diplomatique, } \\
\text { hrvatsko izdanje, veljača } \\
2017 \text {, « Njemačka i izbje- } \\
\text { glice, Kultura dobrodošlice } \\
\text { pred izazovima», Olivier } \\
\text { Cyran, s francuskog preve- } \\
\text { la: Andrea Rudan, pages 3- } \\
5\end{array}$ \\
\hline $\begin{array}{l}\text { Extrait } \\
2\end{array}$ & $\begin{array}{l}\text { Si, en contrôlant le gouvernement } \\
\text { de l'extérieur, le " Rottamatore " } \\
\text { espère [maîtriser l'action] et la } \\
\text { durée de l'administration }\end{array}$ & 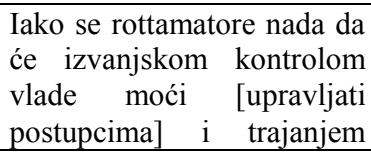 \\
\hline
\end{tabular}




\begin{tabular}{|c|c|c|}
\hline & $\begin{array}{l}\text { Gentiloni tout en se libérant lui- } \\
\text { même pour la campagne qui } \\
\text { s'ouvre, il [met son parti dans } \\
\text { une position difficile] : le PD } \\
\text { devra [assumer encore une fois la } \\
\text { charge] du gouvernement } \\
\text { pendant la campagne et, [comme } \\
\text { l'a relevé] M. D'Alema (La } \\
\text { Repubblica, } 12 \text { décembre 2016), } \\
\text { il pourrait finir " [submergé par } \\
\text { une vague] " aux prochaines } \\
\text { élections, qui [se présentent sur } \\
\text { un plateau d'argent] pour M. } \\
\text { Giuseppe Grillo et ses partisans. } \\
\text { Ces derniers ont d'ailleurs déjà } \\
\text { [revêtu leurs habits favoris], } \\
\text { ceux des défenseurs de la « } \\
\text { dignité des citoyens » contre un } \\
\text { gouvernement et un parti qui } \\
\text { [bafouent la « souveraineté] } \\
\text { populaire» (7). }\end{array}$ & $\begin{array}{l}\text { administracije Paola Genti- } \\
\text { lonija, a pritom sebi } \\
\text { osloboditi prostor za nado- } \\
\text { lazeću kampanju, [svoju } \\
\text { stranku stavlja u težak } \\
\text { položaj]. Naime, Demokra- } \\
\text { tska stranka još će jednom } \\
\text { tijekom kampanje morati } \\
\text { [preuzeti odgovornost] za } \\
\text { vladu i, [kao što je } \\
\text { istaknuo] D’Alema (La } \\
\text { Repubblica, 12. prosinca } \\
\text { 2016.), mogao bi je } \\
\text { [«pomesti val»] na } \\
\text { sljedećim izborima koji za } \\
\text { Grilla i njegove pristaše } \\
\text { [dolaze na srebrnom } \\
\text { pladnju]. Oni su, uostalom, } \\
\text { već navukli svoj najdraži } \\
\text { kostim čuvara «građanskog } \\
\text { dostojanstva» od vlade i } \\
\text { stranaka koje [gaze } \\
\text { «narodni suverenitet] ». }\end{array}$ \\
\hline $\begin{array}{l}\text { Source } \\
2\end{array}$ & $\begin{array}{l}\text { Le Monde diplomatique, janvier } \\
2017 \text {, « Le pari perdu des } \\
\text { sociaux-démocrates italiens } \\
\text { Matteo Renzi se rêve en phénix » } \\
\text { par Raffaele Laudani (Professeur } \\
\text { d'histoire de la pensée politique à } \\
\text { l'université de Bologne), page } 19\end{array}$ & $\begin{array}{l}\text { Le Monde diplomatique, } \\
\text { hrvatsko izdanje, siječanj } \\
2017 \text {, «Izgubljena oklada } \\
\text { Mattea Renzija », Raffaele } \\
\text { Laudani, s francuskog } \\
\text { prevela: Dora Slakoper, } \\
\text { page } 1^{\text {iii }}\end{array}$ \\
\hline
\end{tabular}

Dans un deuxième temps, sur l'échantillon des expressions relevées, nous avons fait une comparaison entre les expressions idiomatiques dans le texte source et leurs correspondances dans le texte cible en nous concentrant sur la transmission du sens contextuel mais aussi sur celui transmis dans la valeur expressive. Il s'agissait donc d'une double comparaison, effectuée au niveau systémique et au niveau textuel. Au niveau du système linguistique, une expression idiomatique de la langue de départ ayant son sens global expressif a été comparée avec une expression (idiomatique ou non) équivalente de la langue cible ayant son sens global expressif ou non expressif. Au niveau textuel, la même expression idiomatique de la langue de départ ayant son sens expressif actualisé en contexte a été comparée avec l'expression (idiomatique ou non) équivalente de la langue cible ayant son sens expressif ou non expressif actualisé en contexte. Cette comparaison consistait ainsi à déchiffrer la valeur expressive comme la valeur contextuelle de chaque expression dans chaque langue, ainsi qu'à confronter la relation entre l'expression française et l'expression équivalente par rapport au texte dans son ensemble. Ce déchiffrage a donné lieu à un accord entre annotateurs pour nous assurer que la grille d'analyse que nous proposons soit la plus objective possible. En effet, comme le déchiffrage repose sur l'intuition des annotateurs, l'objectivité ne peut être atteinte autrement que par un aboutissement à l'accord commun entre annotateurs. Ses précautions prises, l'objectivité atteinte reste néanmoins relative, mais elle nous permet de garantir que nos résultats soient suffisamment significatifs.

\subsection{Les paramètres retenus pour la grille d'analyse}

Nous avons utilisé une grille d'analyse qui consiste à évaluer le transfert des valeurs expressives et des valeurs contextuelles, à indiquer le procédé de transfert employé, si 
l'unité de traduction constitue une expression idiomatique dans la langue cible, si elle dévoile un effet de mise en lumière grâce à l'emploi de l'image et si, au final, elle constitue une équivalence ou correspondance acceptable pour la langue source. Le Tableau 2 résume les paramètres utilisés pour l'évaluation (le chapitre 4.2 offre une explication de ces paramètres dans le cadre de l'analyse des stratégies de traduction qu'ils ont rendue possible).

Tableau 2. Les paramètres de la grille d'analyse

\begin{tabular}{|l|l|}
\hline Nom/code & \multicolumn{1}{|c|}{ Type de paramètre } \\
\hline VE & $\begin{array}{l}\text { valeur expressive (on cherche à évaluer si elle est } \\
\text { transmise) }\end{array}$ \\
\hline VC & $\begin{array}{l}\text { valeur contextuelle (on cherche à évaluer si elle est } \\
\text { transmise) }\end{array}$ \\
\hline EI & $\begin{array}{l}\text { expression idiomatique (on cherche à évaluer s'il } \\
\text { s'agit d'une EI en LC) }\end{array}$ \\
\hline CR & $\begin{array}{l}\text { création (on cherche à évaluer si l'expression en LC } \\
\text { constitue une création) }\end{array}$ \\
\hline Procédé & $\begin{array}{l}\text { procédé de traduction (on cherche à identifier si la } \\
\text { traduction repose sur un calque, une adaptation ou } \\
\text { une modulation) }\end{array}$ \\
\hline Stratégie & $\begin{array}{l}\text { mise à plat, mise en lumière (on cherche à évaluer } \\
\text { la valeur expressive en LC) }\end{array}$ \\
\hline Résultat & $\begin{array}{l}\text { correct, semi-correct, erroné (on évalue le succès de } \\
\text { la traduction) }\end{array}$ \\
\hline
\end{tabular}

Concernant les procédés de traduction, nous nous référons aux procédés généralement admis tels qu'ils ont été définis par Vinay et Darbelnet (1977) et Chuquet et Paillards (1987), sans discuter ici de la classification proposée par ces auteurs (distinguant notamment emprunt, traduction littérale, calque, transposition, modulation, adaptation et équivalence) dans la mesure où elle nous permet d'analyser les deux valeurs, expressive et contextuelle, efficacement. Notre analyse montre que dans le cas des expressions idiomatiques traduite dans le cadre du couple de langue français-croate, on a généralement recours au calque, à l'adaptation et à la modulation. Néanmoins, d'autres paramètres entrent en ligne de compte dans l'analyse des transferts des valeurs expressives et contextuelles.

Notre analyse, présentée dans la section suivante, montre que la traduction des expressions idiomatiques employées présente un grand défi pour les traducteurs.

\section{Résultats}

Nous présentons d'abord les expressions idiomatiques en français avec leurs traductions en croate analysées selon les paramètres permettant de comprendre la stratégie traductionnelle employée. Nous discutons ensuite dans quelle mesure les procédés de traduction connus, tels que le calque, l'adaptation et la modulation relevés dans nos exemples de couples d'expressions français-croate, permettent ou pas de transmettre à la fois le sens expressif et le sens contextuel, et quelles stratégies particulières on observe dans la traduction des expressions idiomatiques dans LMD.

Dans la section 5 consacrée aux Discussions, nous expliquons ce que nos résultats nous apportent comme connaissance sur les problèmes de traduction des expressions idiomatiques du français au croate dans le registre journalistique et sur les stratégies à employer. 


\subsection{Analyse des valeurs expressives et contextuelles des expressions idiomatiques cibles}

Le Tableau 3 montre un échantillon d'expressions idiomatiques en français avec leurs traductions en croate sélectionnées dans LMD et analysées selon la grille d'analyse que nous avons construite pour les besoins de cette étude. Dans l'Annexe, nous offrons la liste des autres couples d'expressions français-croate analysés sur lesquelles se fonde notre étude.

Tableau 3. Analyse des traductions des expressions idiomatiques français-croate selon les paramètres permettant d'identifier les stratégies traductionnelles

\begin{tabular}{|c|c|c|c|c|c|c|c|c|c|}
\hline & Français & Croate & VE & $\mathrm{VC}$ & EI & CR & Procédé & Stratégie & Résultat \\
\hline 1 & $\begin{array}{l}\text { revêtir un } \\
\text { tour }\end{array}$ & $\begin{array}{l}\text { poprimiti } \\
\text { izgled }\end{array}$ & - & + & - & - & adaptation & mise à plat & $\begin{array}{l}\text { semi- } \\
\text { correct }\end{array}$ \\
\hline 2 & $\begin{array}{l}\text { redorer son } \\
\text { blason }\end{array}$ & ulaštiti imidž & + & + & - & + & adaptation & $\begin{array}{l}\text { mise en } \\
\text { lumière }\end{array}$ & correct \\
\hline 3 & $\begin{array}{l}\text { se présenter } \\
\text { sur le } \\
\text { plateau } \\
\text { d'argent }\end{array}$ & $\begin{array}{l}\text { dolaziti na } \\
\text { srebrnom } \\
\text { pladnju }\end{array}$ & + & + & + & - & calque & $\begin{array}{l}\text { mise en } \\
\text { lumière }\end{array}$ & correct \\
\hline 4 & $\begin{array}{l}\text { tailler un } \\
\text { rôle }\end{array}$ & skrojiti ulogu & + & + & - & + & calque & $\begin{array}{l}\text { mise en } \\
\text { lumière }\end{array}$ & correct \\
\hline 5 & $\begin{array}{l}\text { faire de } \\
\text { l'ombre }\end{array}$ & $\begin{array}{l}\text { uzeti najveći } \\
\text { dio }\end{array}$ & - & + & - & - & adaptation & mise à plat & $\begin{array}{l}\text { semi- } \\
\text { correct }\end{array}$ \\
\hline 6 & $\begin{array}{l}\text { tromper } \\
\text { l'ennui }\end{array}$ & $\begin{array}{l}\text { ubijati } \\
\text { dosadu }\end{array}$ & + & + & + & - & adaptation & $\begin{array}{l}\text { mise en } \\
\text { lumière }\end{array}$ & correct \\
\hline 7 & $\begin{array}{l}\text { laisser à } \\
\text { désirer }\end{array}$ & $\begin{array}{l}\text { ne biti uvijek } \\
\text { najbolji }\end{array}$ & - & $-1+$ & - & - & adaptation & mise à plat & correct \\
\hline 8 & $\begin{array}{l}\text { laisser sur le } \\
\text { carreau }\end{array}$ & $\begin{array}{l}\text { tjerati s } \\
\text { pločnika }\end{array}$ & + & $-1+$ & - & + & modulation & $\begin{array}{l}\text { mise en } \\
\text { lumière }\end{array}$ & $\begin{array}{l}\text { semi- } \\
\text { correct }\end{array}$ \\
\hline 9 & $\begin{array}{l}\text { mettre à } \\
\text { rude épreuve }\end{array}$ & testirati & - & + & - & - & adaptation & mise à plat & $\begin{array}{l}\text { semi- } \\
\text { correct }\end{array}$ \\
\hline 10 & peser lourd & $\begin{array}{l}\text { ostaviti } \\
\text { utjecaj }\end{array}$ & - & + & - & - & modulation & mise à plat & $\begin{array}{l}\text { semi- } \\
\text { correct }\end{array}$ \\
\hline 11 & $\begin{array}{l}\text { faire les gros } \\
\text { titres }\end{array}$ & $\begin{array}{l}\text { ne dospi- } \\
\text { jevati na } \\
\text { naslovnice }\end{array}$ & + & + & + & - & adaptation & $\begin{array}{l}\text { mise en } \\
\text { lumière }\end{array}$ & correct \\
\hline 12 & $\begin{array}{l}\text { se jeter sur } \\
\text { l'aubaine }\end{array}$ & $\begin{array}{l}\text { stati na čelo } \\
\text { kampanje }\end{array}$ & - & - & + & - & modulation & mise à plat & erroné \\
\hline 13 & $\begin{array}{l}\text { rester les } \\
\text { bras croisés }\end{array}$ & $\begin{array}{l}\text { ostati } \\
\text { prekriženih } \\
\text { ruku }\end{array}$ & + & + & + & - & calque & $\begin{array}{l}\text { mise en } \\
\text { lumière }\end{array}$ & correct \\
\hline 14 & $\begin{array}{l}\text { briser la } \\
\text { glace }\end{array}$ & razbiti led & + & + & + & - & calque & $\begin{array}{l}\text { mise en } \\
\text { lumière }\end{array}$ & correct \\
\hline 15 & $\begin{array}{l}\text { offrir un } \\
\text { pont d'or }\end{array}$ & $\begin{array}{l}\text { nuditi priliku } \\
\text { koja se ne } \\
\text { propušta }\end{array}$ & - & + & + & - & adaptation & mise à plat & $\begin{array}{l}\text { semi- } \\
\text { correct }\end{array}$ \\
\hline 16 & $\begin{array}{l}\text { prendre la } \\
\text { plume }\end{array}$ & latiti se pera & + & + & + & - & calque & $\begin{array}{l}\text { mise en } \\
\text { lumière }\end{array}$ & correct \\
\hline 17 & $\begin{array}{l}\text { à couper au } \\
\text { couteau }\end{array}$ & $\begin{array}{l}\text { rezati poput } \\
\text { noža }\end{array}$ & + & $-1+$ & - & + & calque & $\begin{array}{l}\text { mise en } \\
\text { lumière }\end{array}$ & $\begin{array}{l}\text { semi- } \\
\text { correct }\end{array}$ \\
\hline
\end{tabular}




\begin{tabular}{|l|l|l|l|l|l|l|l|l|l|}
\hline 18 & $\begin{array}{l}\text { noyé sous } \\
\text { les flots }\end{array}$ & preplavljen & - & + & - & - & adaptation & mise à plat & $\begin{array}{l}\text { semi- } \\
\text { correct }\end{array}$ \\
\hline
\end{tabular}

Nos données étant de taille réduite, nous ne présentons pas d'analyses statistiques mais uniquement les analyses qualitatives. Les données dans le Tableau 3 montrent plusieurs tendances que nous discutons en détail dans les sections suivantes. Le Tableau 4 résume ces différentes tendances dont trois sont récurrentes :

- A consistant à transmettre à la fois les valeurs expressives et contextuelles par recours à une création d'une expression étant donné qu'une expression idiomatique avec ces mêmes valeurs n'est pas disponible dans la langue cible, dont le succès de la traduction peut être optimal ou relatif, illustré par redorer son blason $=>$ ulaštiti imidž,

- B consistant à transmettre à la fois les valeurs expressives et contextuelles par recours à une expression idiomatique existant avec ces mêmes valeurs dans la langue cible, dont le succès de la traduction peut être jugé optimal, illustré par tromper l'ennui $=>$ ubijati dosadu,

- C consistant à transmettre uniquement la valeur contextuelle par recours à une correspondance dans la LC qui n'est pas une expression idiomatique, ni une création, et dons le succès est généralement relatif et parfois optimal, illustré par peser lourd $=>$ ostaviti utjecaj, et deux cas de figure plus rares :

- D consistant à transmettre uniquement la valeur contextuelle par recours à une expression idiomatique dans la LC qui ne permet pas de véhiculer la valeur expressive de l'image d'origine, et dont le succès est relatif et, illustré par offrir un pont d'or => nuditi priliku koja se ne propušta,

- E consistant à recourir à une expression idiomatique qui ne permet pas de transmettre ni la valeur expressive ni contextuelle de l'expression d'origine, et dont le succès est très relatif, illustré par se jeter sur l'aubaine => stati na čelo kampanje.

Tableau 4. Les tendances dans les traductions des expressions idiomatiques français-croate

\begin{tabular}{|c|c|c|c|c|c|c|c|}
\hline & VE & VC & EI & CR & Procédé & Stratégie & Résultat \\
\hline $\mathrm{A}$ & + & + & - & + & $\begin{array}{c}\text { calque ou adaptation ou } \\
\text { modulation }\end{array}$ & mise en lumière & $\begin{array}{c}\text { correct ou semi- } \\
\text { correct }\end{array}$ \\
\hline $\mathrm{B}$ & + & + & + & - & calque ou adaptation & mise en lumière & correct \\
\hline $\mathrm{C}$ & - & + & - & - & adaptation ou modulation & mise à plat & $\begin{array}{c}\text { semi-correct ou } \\
\text { correct }\end{array}$ \\
\hline $\mathrm{D}$ & - & + & + & - & adaptation & mise à plat & semi-correct \\
\hline $\mathrm{E}$ & - & - & + & - & modulation & mise à plat & erroné \\
\hline
\end{tabular}

\subsection{Analyse des stratégies de traduction français-croate employées dans LMD}

Nous discutons dans cette partie les stratégies de traduction que nous avons pu identifier sur la base des traductions des expressions idiomatiques français-croate dans le LMD sélectionnées. Il ne s'agit en aucun cas d'une énumération d'outils, stratégiques en l'occurrence, qui seraient à la disposition des traducteurs. Nous entendons bien que chaque texte a son essence propre et que chaque texte « dirige » les choix du traducteur quant à la recherche de la meilleure des solutions. Notre objectif est plutôt d'observer et décrire les stratégies qui se dévoilent de la grille d'analyses construite dans la section précédente afin de comprendre la place que jouent les expressions idiomatiques dans LMD et, plus particulièrement, leur enjeu dans la traduction. 
Grace à notre grille d'analyse, nous pouvons identifier plusieurs cas de figure : nous pouvons d'abord opposer les cas de figure où les deux valeurs, expressive et contextuelle, ont été transmises à la fois et les cas de figure où une seule des deux valeurs se trouve transférée dans la langue cible au détriment de l'autre. Un seul exemple, présenté à la fin, illustre le cas où aucune des deux valeurs n'a été transmise.

\subsubsection{A la recherche de l'équilibre entre les valeurs expressive et contextuelle}

Nous observons différents types de stratégies permettant de transférer à la fois la valeur expressive et la valeur contextuelle des expressions.

\subsubsection{Calque}

Le calque est un procédé de traduction justifiable lorsque les deux systèmes langagiers se trouvent en parfaite adéquation. C'est le cas des couples d'expressions idiomatiques comme rester les bras croisés $=>$ ostati prekriženih ruku et briser la glace $=>$ razbiti led. Nous remarquons, d'après nos exemples, qu'il s'agit d'expressions idiomatiques dont le sémantisme est à opacité faible, autrement dit à transparence élevée, ce qui rend la traduction par calque applicable sans trop de difficulté. Ce procédé offre une équivalence au niveau formel et sémantique, ainsi la valeur expressive et la valeur contextuelle des expressions idiomatiques sont bel et bien préservées. Néanmoins, on observe dans notre échantillon que le calque n'est pas la solution la plus souvent utilisée, ni possible. On observe également que certains exemples de calque sont des cas de figure particuliers.

Par exemple, la traduction de l'expression prendre la plume $=>$ latiti se pera repose aussi sur le calque puisque littéralement latiti se pera veut dire prendre la plume, et que l'expression croate constitue une expression idiomatique dans cette langue. Néanmoins, on peut remarquer un léger déséquilibre entre les deux langues, dans la mesure où le verbe latiti est un concurrent rare du verbe uzeti qui est le verbe récurent signifiant prendre en croate dans la majorité de contexte. Il s'agit donc, avec latiti, d'un registre de langue différent mais admis pour le style journalistique et qui a un effet expressif plus fort que l'expression idiomatique française. Par conséquent, le déséquilibre en question n'est pas gênant pour la traduction. Au contraire, il est bénéfique puisqu'il soutient le style recherché de LMD.

La transmission des valeurs expressive et contextuelle en recourant au calque peut donner lieu à un autre cas de figure particulier, comme avec tailler un rôle $=>$ skrojiti $u \log u$ où le calque a abouti à une expression qui n'est pas idiomatique en croate. Il y a donc un acte de création, mais le sens reste identique et facile à restituer, comme la valeur expressive grâce à la création.

\subsubsection{Adaptation}

L'adaptation semble être un procédé présentant des solutions bien plus convaincantes que le calque. La majorité des exemples recensés dans le Tableau 3 et en Annexe illustrent les cas d'adaptation. La langue cible, bien qu'offrant une image différente, recourt à des concepts et des référents véhiculant le même sens. Ainsi, nous retrouvons dospijeti na naslovnice (litt. se retrouver sur les couvertures) pour faire les gros titres. Certes, la même image n'a pas été préservée, mais ce qui est bien plus important pour le lecteur l'a été : la valeur expressive et la valeur contextuelle. Le traducteur doit néanmoins porter son attention sur quelques obstacles à éviter. Il ne doit pas tomber dans le piège d'un registre différent qui engendrerait une inadéquation par rapport au texte dans son ensemble ainsi qu'à une déperdition sémantique et prosodique. 
L'adaptation est donc particulièrement efficace quand la LC possède une expression idiomatique propre qui permet d'encoder le même sens que l'expression source. Les images dans les deux langues sont (plus ou moins) différentes, mais la force expressive et le sens sont identiques. C'est le cas aussi avec l'exemple tromper l'ennui => ubijati dosadu (litt. *tuer l'ennui). En effet, les langues portent en elles leur propre culture et donc ont recours à des représentations différentes.

\subsubsection{Modulation}

La transmission des valeurs expressive et contextuelle peut s'obtenir à l'aide d'un autre type de procédé : la modulation. Dans ce cas, on a une transmission fidèle du sens contextuel, tandis que la valeur expressive a été modulée en recourant à la création d'une (nouvelle) image dans la LC, afin de préserver la valeur stylistique de l'expression idiomatique dans la LC. C'est le cas de l'expression redorer son blason => ulaštiti imidž (litt. polir son image/style/look). La stratégie de traduction employée donne un résultat dont l'appréciation demeure d'ordre subjectif, comme dans le cas de toute création, mais cette proposition de traduction constitue une tentative de restituer le sens tout en préservant la force expressive. Le recours à la modulation semble plus rare que le recours à l'adaptation et au calque.

On peut supposer qu'avec le recours à la modulation, la transmission de la valeur expressive sera assurée mais celle de la valeur contextuelle sera moins certaine. En d'autres mots, la modulation permet une transmission partielle de la valeur contextuelle : elle repose sur un éloignement acceptable de la langue source pour restituer au mieux dans la LC un sens en vue des outils linguistiques (in)disponibles en LC. C'est ce qu'on observe avec les exemples expliqués ci-après.

\subsubsection{Eloignement de la valeur contextuelle au profit de la valeur expressive}

Notre analyse montre que lorsque la valeur contextuelle n'est pas respectée «à la lettre », le sens n'est pas pour autant transmis de manière inacceptable. Dans l'ensemble de nos exemples extraits du LMD, il n'y a qu'un seul "faux sens » dans les traductions des expressions idiomatiques, et plusieurs cas où on s'éloigne aussi peu que possible du sens d'origine par soucis de préservation de la valeur expressive. Dans ces exemples, le sens se trouve restitué d'assez près, comme dans à couper au couteau $=>$ koji reže poput noža (litt. qui coupe comme un couteau). Notre analyse indique que ces exemples reposent sur des créations : il semble ainsi s'agir d'une stratégie utile quand dans la LC aucune expression idiomatique n'est disponible. Cette stratégie permet de garder une certaine densité des images, propre du style LMD.

En effet, seule une analyse en contexte peut nous permettre d'évaluer l'effet de la proposition de correspondance en croate :

Français : « Dans le Dracula très statique de Tod Browning, l'acteur Bela Lugosi en offre une vision assez grotesque : blafard, affublé de crocs et d'un accent à couper au couteau, il se meut avec une remarquable lenteur... » (Prolongeau Hubert, " La volupté du sang », Le Monde diplomatique, 2017/2, N 755, p. 27)

Croate: «U statičnom Drakuli Toda Browninga iz 1931. godine, glumac Bela Lugosi djeluje kao veoma groteskna figura: olovne boje lica, s velikim očnjacima i naglaskom koji reže poput noža, kreće se sa zapanjivom sporošću... » (Le Monde diplomatique, 2017/2, $\mathrm{N}^{\circ}$ 50, Literarna slava gospode krvoloka, S francuskog prevela : Marija Čačić, p. 32) 
L'expression française accent à couper au couteau désigne un "accent très prononcé " car «à couper au couteau » renvoie à la notion de densité ou intensité (par analogie à l'expression brouillard à couper au couteau pour désigner « un brouillard très épais »), tandis que la correspondance, traduite littéralement, en croate reste moins évidente à interpréter. En effet, l'expression «naglasak koji reže poput noža » n'est pas idiomatique en croate - ce n'est pas une catachrèse comme en français - si bien que l'image « koji reže poput noža » demeure vivante et invite le lecteur à une interprétation de l'image, à lui attribuer un sens. Le locuteur aura tendance à interpréter l'expression créée par " l'accent qui fait mal aux oreilles, mal comme si on venait de se couper avec un couteau ». On peut considérer que le sens est mieux transmis en recourant à ce type de création que si l'on utilisait une correspondance existante dans la langue mais n'offrant pas d'image, de type $s$ jakim naglaskom (litt. avec un accent très fort). Ce qu'il faut remarquer dans cet exemple est qu'il y a recours au calque pour faire un acte de création étant donné que koji reže poput noža constitue littéralement « qui coupe comme un couteau ».

Il en va de même pour l'expression croate tjerati s pločnika (litt. chasser du trottoir) qui ne révèle pas en soi l'idée d'abandon de laisser sur le carreau, mais propose une interprétation légèrement différente de la réalité par le recours à la modulation et à la création. On peut juger ces traductions comme semi-correctes.

\subsubsection{Eloignement de la valeur expressive au profit de la valeur contextuelle}

Lorsque la seule valeur contextuelle est respectée, on obtient un résultat acceptable mais il $\mathrm{y}$ a une perdition du sens comme dans l'exemple prendre une allure $=>$ biti artikuliran (litt. être articulé). Il y a une «mise à plat» de la valeur expressive de l'expression idiomatique. On peut juger ces traductions comme semi-correctes, même si sur le plan sémantique elles sont tout à fait correctes.

C'est le procédé le plus répandu dans les traductions que nous avons étudiées. La mise à plat ou la banalisation " répond au souci du transfert du sens, mais implique un total irrespect à l'égard du caractère phrasémique de sa forme. » (Le Calvé Ivičević, Vodanović 2017).

Un autre exemple est fourni par l'expression française très répandue laisser à désirer qui devient nisu uvijek najbolji (litt. ne sont pas toujours les meilleurs), où le traducteur a fait le choix de retransmettre la valeur contextuelle, le sens de l'expression figée en contexte. Plus précisément, ne pouvant pas retranscrire la valeur expressive et la valeur contextuelle en une seule et même expression, le traducteur a opté pour la valeur contextuelle. On peut supposer qu'au lieu de réutiliser des figures de style, le traducteur a jugé que le lectorat ne partageait pas les mêmes images et préalables culturelles; il a donc effectué un défigement prescrit par le contexte. Il a recouru à une mise à plat afin de reverbaliser le tout en une expression défigée. En simplifiant le plan de l'expression, le texte traduit a perdu le contexte culturel.

Néanmoins, cette stratégie de traduction est inévitable, puisque les langues française et croate ne présentent pas une symétrie absolue et le traducteur ne peut éviter de se confronter à une absence de lexicalisation dans la langue cible.

Lorsque la seule valeur contextuelle est transmise, on peut avoir des cas de figure différents en fonction du statut de la correspondance dans la LC. Les expressions biti artikuliran et nisu uvijek najbolji ne sont pas des structures idiomatiques en croate. Pour l'exemple offrir un pont d'or, le croate n'admet pas, par exemple, ni une traduction littérale de l'expression ni une expression imagée, mais il offre une expression figée ou collocation : prilika koja se ne propušta (litt. l'opportunité à ne pas laisser passer). Le traducteur a eu donc recours à une mise à plat, mais il a adapté l'expression à la LC en utilisant au mieux ce que qu'elle lui propose. 
La mise à plat peut être également illustrée avec l'exemple mettre à rude épreuve => testirati (litt. tester), où la traduction d'une expression idiomatique imagée par un mot usuel qui ne porte pas de valeur imagé, semble s'éloigner complétement de la valeur expressive originale.

C'est par ces décalages, plus ou moins légers, de la valeur expressive et/ou contextuelle qu'on peut aboutir à un glissement de sens, c'est-à-dire à un faux sens.

\subsubsection{Eloignement de la valeur contextuelle et de la valeur expressive}

C'est le cas de l'exemple se jeter sur l'aubaine => stati na čelo kampanje, qu'on peut apprécier plus facilement dans ses contextes d'apparition :

Français : «Les néonazis du Parti national-démocrate d'Allemagne (NPD) se jettent sur l'aubaine et viennent parader tous les dimanches dans les rues aux côtés de dizaines d'habitants. » (Le Monde diplomatique, 2017/3, N 756, «Un grand élan de solidarité, mais une xénophobie qui progresse ; L'Allemagne et les réfugiés, deux ans après » par Olivier Cyran, pages 12-13)

Croate: «Na čelo kampanje stali su članovi nacističke Nacionaldemokratske stranke (NPD) koji su svake nedjelje organizirali prosvjede uz potporu nekoliko desetaka mještana. » (Le Monde diplomatique, 2017/2, $\mathrm{N}^{\circ}$ 50, « Njemačka i izbjeglice, Kultura dobrodošlice pred izazovima ", Olivier Cyran, S francuskog prevela: Andrea Rudan, p. 5)

La traduction en croate se matérialise dans une expression figée (stati na čelo kampanje litt. se mettre à la tête du partie) dont l'image n'est nullement aussi expressive que celle de l'expression d'origine. De plus, la traduction croate propose une interprétation du sens contextuel plutôt que de le suggérer comme le font les expressions idiomatiques, en prenant le risque de s'éloigner trop du sens initial : se jeter sur l'aubaine réfère à l'idée de «tirer l'avantage, profiter de l'occasion", ce qui confère au sujet (ici les néonazis du Parti national-démocrate d'Allemagne) un trait de caractère particulier, que la traduction en croate n'indique pas.

\section{Discussions}

Dans cette étude, nous avons fait une comparaison entre les textes en français et ceux en croate dans Le Monde diplomatique en nous concentrant sur la transmission du sens contextuel mais aussi sur celui transmis dans la valeur expressive. Notre analyse démontre que les expressions idiomatiques constituent un véritable défi pour les traducteurs. Ces expressions dotées d'une force expressive font non seulement partie du style du récit mais sont aussi porteuses de sens. Le but de notre recherche a donc été d'analyser les correspondances linguistiques que présentent les deux systèmes étudiés quant aux expressions idiomatiques ainsi que leurs équivalences discursives.

La traduction des articles de LMD exige un type spécifique de médiation interlinguistique qui sous-entend une adaptation du texte source au lectorat cible, qu'il soit croate, anglais ou italien par exemple. Le procès d'adaptation/adéquation inclut tout particulièrement les expressions stylistiques parmi lesquelles les expressions idiomatiques dont l'interprétation peut dépendre de la situation de communication. Très souvent le sens de la phrase ne peut pas être compris sans procéder à un décodage des expressions idiomatiques, ce qui amène le traducteur à leur accorder une plus grande importance par rapport aux autres unités lexicales. 
Le Monde diplomatique étant un journal revendiquant son héritage littéraire, nous considérons que les expressions idiomatiques représentent une force expressive, discursive qui exprime aussi un sens. Les expressions idiomatiques n'ont pas une valeur nulle mais présentent bien des valeurs expressive, contextuelle, littéraire, stylistique, discursive qu'il faut prendre en compte. C'est pourquoi nous proposons d'avoir recours à une compensation lorsque la langue cible ne permet pas une traduction linguistique et sémantico-contextuelle adéquate. Si le croate ne permet pas une véritable équivalence sur le plan expressif et contextuel, le traducteur devra-t-il alors remédier à cette perte autre part dans le texte, là où sa langue le lui permet.

Les expressions idiomatiques portent en elles cet éternel dilemme de la dualité de la forme et du contenu. Elles endossent « [...] deux sens, un sens compositionnel ou littéral, qui n'est pas le leur, et un sens qui est le leur, qui s'avère non compositionnel et qui donc est dit opaque ou figé, sens lexicalisé ou conventionnel, qui doit être appris comme étant le sens de la dénomination idiomatique [...] » (Kleiber 2010 :139). Ici, la difficulté pour le traducteur repose dans le fait que le sens global et non-compositionnel de l'expression figée n'est jamais complètement affranchi de l'image compositionnelle rattachée à celle-ci. D'autre part, la valeur contextuelle des expressions idiomatiques est porteuse de sens puisque le traducteur traduit un texte, l'activité traduisante étant une activité discursive et interprétative. Le sens global et lexicalisé des expressions idiomatiques va être parfois réactualisé en contexte. Il ne s'agit donc pas pour le traducteur de traduire uniquement la valeur contextuelle ou discursive de de ces expressions mais aussi leur valeur expressive.

Rares sont les situations de parfaite adéquation entre deux systèmes langagiers et c'est bien le cas du couple franco-croate. Le traducteur ne peut pas toujours préserver les deux aspects qui vont de paires dans les expressions idiomatiques, comme la double face d'une monnaie de pièce. Or, le traducteur ne sait faire autre que de traduire quand même. Comme le soulignent Le Calvé Ivičević et Vodanović (2017) « la superposition du sens lexicalisé avec l'image surgissant du sens littéral pose la question de l'équivalence sémantique ainsi [...] que celle de l'équivalence stylistique, ce qui invite dans tous les cas à la recherche d'une image comparable ». D'autre part, nous sommes tous d'accord sur le fait que l'on traduit une langue-culture, et les expressions idiomatiques sont ancrées dans la culture propre à une langue. C'est pourquoi le traducteur se heurtera dans la grande majorité des cas à un processus de renonciation. Il devra donc, comme le dit Eco, négocier : « la traduction se fonde sur des processus de négociation, cette dernière étant justement un processus selon lequel, pour obtenir quelque chose, on renonce à quelque chose d'autre, et d'où, au final, les parties en jeu sortent avec un sentiment de satisfaction raisonnable et réciproque, à la lumière du principe d'or selon lequel on ne peut pas tout avoir. » (Eco, $2006: 18)$.

\section{Conclusion}

Notre étude aborde la problématique du rôle joué par des expressions idiomatiques dans Le Monde diplomatique et de l'importance de la prise en compte de ce rôle dans la traduction. Les expressions idiomatiques constituent un élément caractéristique du style journalistique et, dans Le Monde diplomatique, elles participent également au maillage entre le style littéraire, revendiqué par le journal, et le style journalistique propre au journal. Ces expressions, dotées d'une force expressive, font non seulement partie du style du journal mais sont aussi porteuses de sens. Comme le journal est traduit dans 22 langues, les expressions figées idiomatiques posent des problèmes de traduction, notamment dans les langues qui ne partagent pas une symétrie forte dans l'expression des images, comme le couple de langue français-croate.

Pour réaliser notre recherche, nous avons utilisé le corpus parallèle franco-croate du journal Le Monde diplomatique dont les textes abordent des sujets politiques actuels. Nous 
avons relevé 38 couples d'expressions idiomatiques que nous avons analysés du point de vue des stratégies de traduction employées et du point de vue de l'adéquation entre les deux sens, expressif et contextuel. Notre analyse des paires français-croate des expressions idiomatiques figées montre que les stratégies de traduction permettant de transmettre les deux valeurs sont plus rares et que dans la majorité de cas une valeur est privilégiée au détriment de l'autre. Notre analyse montre aussi que les erreurs, soit des cas de figure où aucune valeur n'est transmise, sont extrêmement rares.

Nous émettons l'hypothèse que, si une langue ne permet pas une équivalence sur le plan expressif et contextuel des expressions idiomatiques employées dans le texte source, la stratégie de traduction à mettre en place pourrait consister en une remédiation à cette perte en recourant aux expressions idiomatiques autre part dans le texte, là où la langue cible le permet. Cette remédiation pourrait également consister à recourir à des références culturelles admises par le genre et adapté au public cible là où les textes se prêtent particulièrement bien à ce type d'ajout. Bien évidemment, ces recommandations plaident pour une traduction de type « cibliste », au lieu de « sourcier» (cf. Ladmiral 2014). D'une manière plus générale, nos résultats mettent en évidence l'utilité de ce type de recherches interdisciplinaires pour une approche interculturelle du processus de traduction, qui reflète bien des divergences de nature conceptuelle, linguistique et socioculturelle entre les langues comparées.

\section{Références bibliographiques}

Arčan, M. (2018). A comparison of Statistical and Neural MT for Slovene, Serbian and Croatian. Fišer, D., Pančur, A. (éd.). Proceedings of the Conference on Language Technologies \& Digital Humanities, Ljubljana : Ljubljana University Press, Faculty of Arts. pp. 3-11.

Bally, Ch. (1926). Le langage et la vie. Paris : Payot.

Benson, M., Benson E., Ilson, R. (1997). The BBI Dictionary of English Word Combinations, 2nd éd. Amsterdam/Philadelphia : John Benjamins.

Ben Amor, Th. (2008). Défigement et traduction intralinguale et interlinguale. Meta, 53(2), pp. 443455.

Burlot, F., Yvon, F. (2017). Évaluation morphologique pour la traduction automatique : adaptation au français. Proceedings of the Second Conference on Machine Translation. Copenhagen: Association for Computational Linguistics. pp. 43-55.

Charaudeau, P. (2005). Les médias et l'information. L'impossible du discours. Bruxelles : De Boeck Université.

Chuquet, H., Paillard, M. (1987). Approche linguistique des problèmes de traduction anglaisfrançais. Publication Gap : Ophrys.

Curea, A. (2008). L'expressivité linguistique : un objet problématique dans la théorie de Charles Bally. In Durand J. Habert B., Laks B. (éds.) Congrès Mondial de Linguistique Française CMLF'08, Histoire, épistémologie, réflexivité. Paris : Institut de Linguistique Française, 917-928.

Dubois, M.-M. (1973). Dictionnaire anglais-français des locutions et expressions verbales. Paris : Larousse.

Durieux, Ch. (2009). Vers une théorie décisionnelle de la traduction. Lisa, 7/3, pp. 349-367.

Durieux, Ch. (2008). Mettre la main sur le figement lexical : la démarche du traducteur. Meta, 53(2), pp. 324-332.

Eco, U. (2006). Dire presque la même chose - Expériences de traduction. Paris : Editions Grasset et Fasquelle.

Gambier, Y. (2006). Transformations in international news. Conway, K., Bassnett, S. (éds.). Translation in Global News. Coventry : University of Warwick. pp. 9-21.

Gläser, R. (1988). The grading of idiomaticity as a presupposition for a taxonomy of idioms. Hüllen, W., Schulze, R. (éds.). Understanding the Lexicon. Tübingen : De Gruyter. pp. 264-279.

Gonzales Rey, I. (2002). La phraséologie du français. Toulouse : Presses universitaires du Mirail.

Gross, G. (1996). Les expressions figées en français : noms composés et autres locutions. Paris : Ophrys. 
Haquin, Y. (2015). Approche linguistique et traductologique du double sens en phraséologie. Pélage, C., Fasquel, S., Natanson, B. Double(s) sens : Espagne-Amérique Latine, Orléans : Paradigmes, pp. 39-53.

Jacques, M.-P., Tutin, A. (éds.). (2018). Lexique transversal et formules discursives des sciences humaines. Londres : ISTE Editions.

Jernej, J. (1992). O klasifikaciji frazema. Filologija, 20-21, pp. 191-197.

Kleiber, G. (2010). Le parcours du sens : d'une langue a l'autre. Meta, 55(1), pp. 136-146.

Ladmiral, J.-R. (2014). Sourcier ou cibliste. Les profondeurs de la traduction. Paris: Les Belles lettres.

Ladmiral, J.-R., Lipiansky, E.M. (2015). La Communication interculturelle. Paris : Les Belles Lettres, (1989 Armand Colin).

Laurian, A.-M. (éd.). (2004). Dictionnaires bilingues et interculturalité. Berne : Peter Lang.

Laurian, A.-M. (2004). Introduction. Problématiques générales des dictionnaires bilingues : du lexique à la culture. Laurian, A.-M. (éd.). Dictionnaires bilingues et interculturalité. pp. 1-13.

Le Calvé Ivičević, E., Vodanović, B. (2017). Des vertes et des pas mures : fruits et légumes dans les phrasemes français et croates. Палимпсест/Palimpsest. International Journal for Linguistic, Literary and Cultural Research, II, 3, pp. 51-61.

Le Bel, E. (2006). Traduire la phraséologie : réflexions méthodologiques et étude de cas. RøL 5, pp. 57-70.

Ljubešić, N., Bago, P., Boras, D. (2010). Statistical machine translation of Croatian weather forecast: How much data do we need? Luzar-Stiffler, V., Jarec, I., Bekic, Z. (éds). Proceedings of the ITI 2010 32nd International Conference on Information Technology Interfaces. Zagreb: SRCE University Computing Centre. pp. 91-96.

Maillot, J. (1984). Expressions idiomatiques en anglais et en français. Traduire 119, pp. 7-11.

Mejri, S. (1997). Le figement lexical : descriptions linguistiques et structuration sémantique. Tunis : Publications de la Faculté des lettres de Manouba.

Mejri, S. (2008). Figement et traduction : problématique générale. Meta, 53 (2), pp. 244-252.

Misri, G. (1990). La traduction des expressions figées. Lederer, M. (éd). Etudes traductologiques en hommage à D. Seleskovitch. Paris : Minard. pp. 143-163.

Mogorrón Huerta, P. (2008). Compréhension et traduction des locutions verbales. Meta, 53(2), pp. 378-406.

Pecman, M. (2007). L'enjeu de la classification en phraséologie. Häcki Buhofer, A., Burger, H. (éds.). Phraseology in Motion II. Theorie und Anwendung. Akten der Internationalen Tagung zur Phraseologie, Basel : Schneider Hohengehren Verlag. pp. 29-47.

Rey, A., Chantreau, S. (1994). Dictionnaire des expressions et locutions. Paris : Robert.

Saussure, F. de (1969). Cours de linguistique générale. Paris : Payot.

Seleskovitch, D., Lederer, M. (1984). Interpréter pour traduire. Paris : Didier Erudition. pp. 294-308.

Sesar, D. (1998). O mjestu i ulozi krilatica u frazeološkim rječnicima. Filologija, 30-31, pp. 305-312.

Vaguer, C. (2011). Expressions figées et traduction : langue, culture, traduction automatique, apprentissage, lexique. Anscombre, J.Cl., Mejri, S. Le figement linguistique : la parole entravée, Paris : Honoré Champion, pp. 391-411.

Vinay, J.-P., Darbelnet, J. (1977). Stylistique comparée du français et de l'anglais : méthode de traduction. Nouvelle édition revue et corrigée [avec une bibliogr. augmentée]. Paris : Didier.

Xatara, C. M. (2002). La traduction phraséologique. Meta, 47(3), pp. 441-444.

Zinglé, H., Brobeck-Zingle, M.-L. (2003). Dictionnaire combinatoire du français. Expressions, locutions et constructions. Paris : La Maison du dictionnaire.

\footnotetext{
${ }^{\mathrm{i}}$ Le site du journal Le Monde Diplomatique : https://www.monde-diplomatique.fr/diplo/apropos

${ }^{\text {ii }}$ La version croate de LMD est disponible sur la page : https://lemondediplomatique.hr

iii On remarque, par ailleurs, que cet article a été déplacé de la page 19 dans la version originale à la première page dans la version croate, sans doute car le sujet porte sur la vie politique italienne qui est généralement suivie de près par les lecteurs croates.
}

\section{Annexe}


La liste complémentaire des couples d'expressions français-croate analysées selon la grille d'analyse construite pour les besoins de la présente étude.

\begin{tabular}{|c|c|c|c|c|c|c|c|c|c|}
\hline & Français & Croate & VE & $\mathrm{VC}$ & EI & $\mathrm{CR}$ & Procédé & Stratégie & Résultat \\
\hline 19 & $\begin{array}{l}\text { prendre une } \\
\text { allure }\end{array}$ & biti artikuliran & - & + & - & - & adaptation & $\begin{array}{l}\text { mise à } \\
\text { plat }\end{array}$ & $\begin{array}{l}\text { semi- } \\
\text { correct }\end{array}$ \\
\hline 20 & serrer la vis & $\begin{array}{l}\text { uspostaviti } \\
\text { oštrije mjere }\end{array}$ & - & + & + & - & adaptation & $\begin{array}{l}\text { mise à } \\
\text { plat }\end{array}$ & $\begin{array}{l}\text { semi- } \\
\text { correct }\end{array}$ \\
\hline 21 & $\begin{array}{l}\text { emboîter le } \\
\text { pas }\end{array}$ & $\begin{array}{l}\text { podupirati u } \\
\text { tome }\end{array}$ & - & + & - & - & adaptation & $\begin{array}{l}\text { mise à } \\
\text { plat }\end{array}$ & $\begin{array}{l}\text { semi- } \\
\text { correct }\end{array}$ \\
\hline 22 & $\begin{array}{l}\text { (ne pas) } \\
\text { sauter aux } \\
\text { yeux }\end{array}$ & teško uočiti & - & + & - & - & adaptation & $\begin{array}{l}\text { mise à } \\
\text { plat }\end{array}$ & $\begin{array}{l}\text { semi- } \\
\text { correct }\end{array}$ \\
\hline 23 & $\begin{array}{l}\text { ne pas payer } \\
\text { de mine }\end{array}$ & $\begin{array}{l}\text { izgledati pitomo } \\
\text { na prvi pogled }\end{array}$ & - & + & - & + & adaptation & $\begin{array}{l}\text { mise à } \\
\text { plat }\end{array}$ & $\begin{array}{l}\text { semi- } \\
\text { correct }\end{array}$ \\
\hline 24 & $\begin{array}{l}\text { passer aux } \\
\text { actes }\end{array}$ & $\begin{array}{l}\text { prijeći s riječi na } \\
\text { djela }\end{array}$ & + & + & + & - & adaptation & $\begin{array}{l}\text { mise en } \\
\text { lumière }\end{array}$ & correct \\
\hline 25 & $\begin{array}{l}\text { faire mine de } \\
\text { (ne pas) }\end{array}$ & tvrditi & - & + & - & - & adaptation & $\begin{array}{l}\text { mise à } \\
\text { plat }\end{array}$ & $\begin{array}{l}\text { semi- } \\
\text { correct }\end{array}$ \\
\hline 26 & $\begin{array}{l}\text { faire tache } \\
\text { d'huile }\end{array}$ & širiti se & - & + & - & - & adaptation & $\begin{array}{l}\text { mise à } \\
\text { plat }\end{array}$ & $\begin{array}{l}\text { semi- } \\
\text { correct }\end{array}$ \\
\hline 27 & $\begin{array}{l}\text { prendre } \\
\text { ombrage }\end{array}$ & $\begin{array}{l}\text { sumnjičavo } \\
\text { ukazati }\end{array}$ & - & + & - & - & adaptation & $\begin{array}{l}\text { mise à } \\
\text { plat }\end{array}$ & $\begin{array}{l}\text { semi- } \\
\text { correct }\end{array}$ \\
\hline 28 & $\begin{array}{l}\text { dessiner les } \\
\text { contours }\end{array}$ & ukazati na & - & + & - & - & adaptation & $\begin{array}{l}\text { mise à } \\
\text { plat }\end{array}$ & $\begin{array}{l}\text { semi- } \\
\text { correct }\end{array}$ \\
\hline 29 & $\begin{array}{l}\text { partir en } \\
\text { croisade }\end{array}$ & povesti rat & + & + & + & - & adaptation & $\begin{array}{l}\text { mise en } \\
\text { lumière }\end{array}$ & correct \\
\hline 30 & $\begin{array}{l}\text { rogner sur } \\
\text { tout }\end{array}$ & $\begin{array}{l}\text { srezati sve } \\
\text { troškove }\end{array}$ & + & + & + & - & adaptation & $\begin{array}{l}\text { mise en } \\
\text { lumière }\end{array}$ & correct \\
\hline 31 & $\begin{array}{l}\text { changer la } \\
\text { donne }\end{array}$ & $\begin{array}{l}\text { promijeniti } \\
\text { stvari }\end{array}$ & - & + & - & - & adaptation & $\begin{array}{l}\text { mise à } \\
\text { plat }\end{array}$ & $\begin{array}{l}\text { semi- } \\
\text { correct }\end{array}$ \\
\hline 32 & $\begin{array}{l}\text { faire tourner } \\
\text { la machine }\end{array}$ & pokrenuti & - & + & - & - & adaptation & $\begin{array}{l}\text { mise à } \\
\text { plat }\end{array}$ & $\begin{array}{l}\text { semi- } \\
\text { correct }\end{array}$ \\
\hline 33 & $\begin{array}{l}\text { être ouvert à } \\
\text { tous les vents }\end{array}$ & $\begin{array}{l}\text { biti otvoren sa } \\
\text { svih strana za } \\
\text { sve }\end{array}$ & - & + & - & - & adaptation & $\begin{array}{l}\text { mise à } \\
\text { plat }\end{array}$ & $\begin{array}{l}\text { semi- } \\
\text { correct }\end{array}$ \\
\hline 34 & $\begin{array}{l}\text { être à somme } \\
\text { nulle }\end{array}$ & igra bez rizika & + & + & + & - & adaptation & $\begin{array}{l}\text { mise en } \\
\text { lumière }\end{array}$ & correct \\
\hline 35 & $\begin{array}{l}\text { regarder } \\
\text { passer les } \\
\text { trains }\end{array}$ & $\begin{array}{l}\text { bespomoćno } \\
\text { stajati po strani }\end{array}$ & - & + & + & - & adaptation & $\begin{array}{l}\text { mise en } \\
\text { lumière }\end{array}$ & $\begin{array}{l}\text { semi- } \\
\text { correct }\end{array}$ \\
\hline 36 & tenir tête & suprotstaviti se & - & + & - & - & adaptation & $\begin{array}{l}\text { mise à } \\
\text { plat }\end{array}$ & $\begin{array}{l}\text { semi- } \\
\text { correct }\end{array}$ \\
\hline 37 & $\begin{array}{l}\text { gagner du } \\
\text { terrain }\end{array}$ & širiti se & - & + & - & - & adaptation & $\begin{array}{l}\text { mise à } \\
\text { plat }\end{array}$ & $\begin{array}{l}\text { semi- } \\
\text { correct }\end{array}$ \\
\hline 38 & $\begin{array}{l}\text { rebrousser } \\
\text { chemin }\end{array}$ & odlučiti se & - & + & - & - & adaptation & $\begin{array}{l}\text { mise à } \\
\text { plat }\end{array}$ & $\begin{array}{l}\text { semi- } \\
\text { correct }\end{array}$ \\
\hline
\end{tabular}

